\section{The influence of sentence}

context and accented speech on lexical access in

\section{second-language auditory word recognition*}

\author{
EVELYNE LAGROU \\ ROBERT J. HARTSUIKER \\ WOUTER DUYCK \\ Ghent University
}

(Received: January 26, 2012; final revision received: June 13, 2012; accepted: July 20, 2012; first published online 27 September 2012)

\begin{abstract}
Until now, research on bilingual auditory word recognition has been scarce, and although most studies agree that lexical access is language-nonselective, there is less consensus with respect to the influence of potentially constraining factors. The present study investigated the influence of three possible constraints. We tested whether language nonselectivity is restricted by (a) a sentence context in a second language (L2), (b) the semantic constraint of the sentence, and (c) the native language of the speaker. Dutch-English bilinguals completed an English auditory lexical decision task on the last word of low-and high-constraining sentences. Sentences were pronounced by a native Dutch speaker with English as the L2, or by a native English speaker with Dutch as the L2. Interlingual homophones (e.g., lief "sweet" - leaf /liff) were always recognized more slowly than control words. The semantic constraint of the sentence and the native accent of the speaker modulated, but did not eliminate interlingual homophone effects. These results are discussed within language-nonselective models of lexical access in bilingual auditory word recognition.
\end{abstract}

Keywords: bilingualism, sentence context, accented speech, auditory word recognition

During the last decades, much research on bilingual word recognition has focused on the question whether lexical access is language-selective or not. By now, there is evidence from the visual (e.g., Dijkstra \& Van Heuven, 1998; Duyck, 2005; Duyck, Van Assche, Drieghe \& Hartsuiker, 2007; Van Assche, Duyck, Hartsuiker \& Diependaele, 2009), and to a much lesser extent, from the auditory domain (e.g., Ju \& Luce, 2004; Lagrou, Hartsuiker \& Duyck, 2011; Marian \& Spivey, 2003; Schulpen, Dijkstra, Schriefers \& Hasper, 2003; Spivey \& Marian, 1999; Weber \& Cutler, 2004) in favor of a language-nonselective account of lexical access. According to this account, lexical representations from both lexicons are activated at least to a certain degree during word recognition, even when only one language is task-relevant. It is less clear, however, whether there are factors that can constrain language-nonselective lexical access, such as the context of the to-be-recognized words. In the visual domain, a few studies have recently addressed this question (Duyck et al., 2007; Libben \& Titone, 2009; Schwartz \& Kroll, 2006; Titone, Libben, Mercier, Whitford \& Pivneva, 2011; Van Assche et al., 2009; Van Assche, Drieghe, Duyck, Welvaert \& Hartsuiker, 2011;

\footnotetext{
* This research was made possible by the Research Foundation-Flanders (FWO) of which Evelyne Lagrou is a research fellow.
}

Van Hell \& de Groot, 2008), whereas such evidence is almost completely lacking for the auditory domain. In the present study, we therefore investigated whether the auditory presentation of a meaningful sentence context is a factor that can constrain lexical access to the currently relevant lexicon. Moreover, we examined whether the semantic predictability of target words in the sentence is a restricting factor, and additionally, we investigated the influence of sub-phonemic cues, inherent to the native accent of the speaker, on parallel language activation.

\section{Bilingual word recognition in isolation}

Evidence for language-nonselective lexical access in bilingual auditory word recognition was first reported by Marian and colleagues. ${ }^{1}$ In the eyetracking study of Spivey and Marian (1999), late Russian-English bilinguals who were very proficient in their L2 and living

\footnotetext{
1 Although the instructions that participants received in the studies by Marian and colleagues and by Weber and Cutler (2004) actually consist of more than one word, we nevertheless considered these studies as isolation studies. We did this because the (very short) preceding sentences in these studies were identical across trials, and hence characterized by the same syntactic structure, lacking any semantic variation (i.e., "Click on the....").
} 
in an L2-dominant environment, were instructed in their L2 to pick up a real-life object (e.g., Pick up the marker). The participants fixated more on competitor items with a name in the irrelevant L1 that was phonologically similar to the target (e.g., a stamp; marka in Russian) than on distracter objects with a name in L1 that was phonologically unrelated to the L2 target. Additionally, there was evidence for language nonselectivity even when participants were listening in L1. When Russian-English bilinguals received an instruction like: Podnimi marku "Pick up the stamp", they looked more often to interlingual competitor objects (marker) than to distracter objects. Analogous to the findings with the English instructions, this can be explained because the English translation equivalent of marka, stamp, is more phonologically similar to the Russian target word marku than to the distracters.

These results were partly replicated by Weber and Cutler (2004) in a later study with Dutch-English bilinguals. These bilinguals, who were living in an L1-dominant environment, were instructed to click on one of four pictures presented in a display, and move it to another location on the computer screen (e.g., Pick up the desk and put it on the circle). There were more fixations on competitor objects whose name had a phonetically similar L1 onset than to distracter objects (e.g., when instructed to pick up the desk, there were more fixations on a picture of a lid than on control items, because lid is the translation equivalent of the Dutch word deksel, phonologically overlapping with the L2 target desk). However, when these participants were instructed in their L1 (e.g., target deksel) competitor items (desk) were not fixated longer than control items, which suggests that nontarget language representations in L2 are not activated strongly enough to influence $\mathrm{L} 1$ recognition.

In one of Schulpen et al.'s (2003) experiments, DutchEnglish bilinguals completed a cross-modal priming task in which primes were presented auditorily and targets visually. Visual lexical decision times were longer when the target was preceded by an interlingual homophone than when the target was preceded by a monolingual control. For instance, responses after the pair /liss/ - LEASE were slower than after /frem/ - FRAME (/lis/ is the Dutch translation equivalent for groin). The observation of longer reaction times after interlingual homophone pairs suggested that bilinguals activated both the Dutch and the English meaning of the homophone. Furthermore, the authors observed that the auditory presentation of the English pronunciation of the interlingual homophone led to faster decision times on the related English target word than the Dutch version of the interlingual homophone. This indicates that these subtle differences between homophones may affect the degree of crosslingual activation spreading, which will turn out to be important for the present study. These differences are most likely situated at the sub-phonemic level (e.g., languages often differ in the length of voice onset time (VOT)), but it is possible that there are suprasegmental differences too (e.g., Lee \& Nusbaum, 1993).

Further studies on the influence of sub-phonemic cues on lexical access in bilinguals were reported by Lagrou et al. (2011) and Ju and Luce (2004). Lagrou et al. conducted a lexical decision experiment in L2 or L1 with Dutch-English bilinguals, living in an L1-dominant environment. The participants responded more slowly to homophones (e.g., lief "sweet" - leaf /liff/) than to matched control words, both in L2 and L1, whereas a monolingual English control group showed no effect. Moreover, this study investigated whether the listener's selectivity of lexical access is influenced by the speaker's L1. With this aim, targets were pronounced by a native Dutch speaker with English as the L2 or by a native English speaker with Dutch as the L2. Although the speaker's accent contains language cues that might affect the activation of target and non-target languages (Schulpen et al., 2003), there was no interaction between the homophone effect and the native language of the speaker. In sum, the results of this study suggest that bilinguals do not use these language- and speaker-specific sub-phonemic cues to restrict lexical access to only one lexicon, even though this implies a less efficient strategy for lexical search.

Ju and Luce (2004) also found evidence for languagenonselective lexical access. Here however, the effect was modulated by sub-phonemic information related to language-specific voice-onset times (VOTs). In a visual world eye-tracking study, Spanish-English bilinguals fixated pictures of interlingual competitors (nontarget pictures whose English names (e.g., pliers) shared a phonological similarity with the Spanish targets (e.g., playa "beach")) more frequently than control distracters. However, this effect was only found when the Spanish target words were altered to contain English-appropriate voice onset times. When the Spanish targets had Spanish VOTs, no L1 interference was found. The results of this study suggest that bilingual listeners may still use finegrained, sub-phonemic, acoustic information related to language-specific VOT to regulate cross-lingual lexical activation. At first sight, this is in contrast with the result of Lagrou et al. (2011). However, in the $\mathrm{Ju}$ and Luce study, a salient acoustic feature (voicing) was manipulated systematically, so that this artificial cue was a reliable and consistent predictor of language membership, whereas the stimuli in the Lagrou et al. study differed on a wider range of acoustic parameters (i.e., all subphonemic cues related to the native accent of the speaker). Moreover, in the study of Ju and Luce all stimuli started with voiceless stops, whereas the stimuli of Lagrou et al. started with a variety of sounds (i.e., nasals and fricatives). 


\section{Bilingual word recognition in a sentence context}

Monolingual studies have demonstrated that contextual information is used to facilitate word recognition in the native language. For example, when reading ambiguous words, context helps to select the correct interpretation (e.g., Binder \& Rayner, 1998; Onifer \& Swinney, 1981; Rayner \& Frazier, 1989). Moreover, predictable words are processed faster than non-predictable words (e.g., Schwanenflugel \& LaCount, 1988; Schwanenflugel \& Shoben, 1985; Stanovich \& West, 1983). Semantic information provided by sentence context may also influence lexical selection in bilingual visual word recognition. For example, Schwartz and Kroll (2006) and Van Hell and de Groot (2008), found that the cognate facilitation effect (i.e., faster RTs on cognates than on matched control items) and the homograph effect (i.e., slower RTs on interlingual homographs than on matched control items), markers of cross-lingual lexical interactions in the visual domain, were annulled or diminished when reading high-constraining sentences. In the study by Van Assche et al. (2011), crosslingual interactions in high-constraining sentences were significant, both on the early and late reading times, whereas in a study by Libben and Titone (2009), this was only the case on the early reading time measures and not on the late comprehension measures. According to Titone et al. (2011), a possible explanation for the differences between studies could be that bilinguals differ in the relative degree of L2 proficiency or other variables that were not taken into account. Taken together, these studies indicate that semantic constraint influences, but does not annul, the co-activation of representations from both languages in the bilingual lexicon, at least in visual language processing.

In the auditory domain, research on bilingual word recognition in a sentence context is more scarce. Chambers and Cooke (2009) investigated whether interlingual lexical competition is influenced by the prior sentence context. In this visual world study, EnglishFrench bilinguals with varying proficiency levels listened to L2 sentences, and were instructed to click on the image that represented the sentence-final word. Each display contained an image of the final noun target (e.g., chicken), an interlingual near-homophone (e.g., pool) whose name in English is phonologically similar to the French target (e.g., poule "chicken"), and two unrelated distracter items. The interlingual competitors were fixated more than unrelated distracter items when the prior sentence information was compatible with the competitor (i.e., both the French target and the interlingual near-homophone are plausible in the sentence context, e.g., Marie va décrire la poule "Marie will describe the chicken"), but not when this sentence information was incompatible with the competitor (i.e., only the French target, but not the interlingual near-homophone is plausible in the sentence context, e.g., Marie va nourrir la poule "Marie will feed the chicken"). These findings suggest that semantic constraints imposed by a sentence context may override activation of non-target language lexical competitors in the auditory domain.

FitzPatrick and Indefrey (2010) recorded EEGs from Dutch-English bilinguals listening to sentences in L2, containing semantic incongruities that typically elicit an N400 component. When listening to an incongruity in L2, this component is delayed in comparison with the component when listening to an incongruity in L1. In one condition of this study, the last word of the sentence was a word with initial overlap with an L1 translation equivalent of the most probable sentence completion (e.g., "My Christmas present came in a bright-orange doughnut" (initial overlap with "doos" where doos is Dutch for box). There was an N400 effect to L1 translation equivalents that were initially congruent with the sentence context. Importantly, this N400 had the same timing as the N400 in response to a semantic incongruity whose translation equivalent did not have initial congruence. Thus, when listening to sentences in L2, L1 competitors were not activated (or these L1 competitors are at least not considered for semantic integration). Because these sentences are quite semantically constraining towards the targets, FitzPatrick and Indefrey argued that sentences that bias towards specific lexical representations in the target language yield no cross-lingual effects.

Although both studies above used meaningful sentences in their studies, there have been no bilingual studies on auditory word recognition that directly manipulated the degree of semantic sentence constraint within-study, assessing its influence on cross-lingual interactions. The results of Chambers and Cooke (2009) and FitzPatrick and Indefrey (2010) suggest that contextual factors may have a larger impact on the degree of language selectivity in the auditory domain than in visual word recognition. However, it may also be the case that modulations by semantic constraint may be more pronounced for words with interlingual form overlap only (i.e., homographs and homophones, as used here) than for the typical cognates (e.g., Van Assche et al., 2011) in the visual studies, because such a constraint is compatible with the (shared) meaning of the L1 and L2 reading of cognates but only compatible with one of the two readings of a homograph/homophone. As such, a suggested interaction between sentence constraint and modality (visual vs. auditory) may be a by-product of the type of critical stimuli used to assess cross-lingual interactions. For the auditory domain, it remains possible that under high constraint, only one homophone meaning is considered, rendering the stimulus similar to one without form overlap. 


\section{The present study}

Our goal was to address three questions. First, we investigated whether there is parallel language activation when listening to meaningful sentences in L2. Second, we investigated the influence of semantic constraint on lexical access when listening in L2. Third, we also tested whether sub-phonemic cues, provided by the native accent of a speaker, are used to restrict lexical access when listening to sentences. Our previous study (Lagrou et al., 2011) suggested that these sub-phonemic cues inherent to the native accent of the speaker are not used to restrict lexical access to the currently relevant lexicon when listening to words in isolation. However, in daily life people usually do not listen to isolated words, but have conversations with other people. Of course, a continuous stream of auditory input contains far more cues that could provide the listener with information about the language in use, which makes it more likely that such cues are indeed used to restrict lexical access when the input consists of sentences compared to isolated words. On the other hand, both in real life and in our experiments, speakers sometimes speak in a language that is the L2 to them, so that the cues picked up from the speaker's accent may be misleading with respect to language membership. Because cues based on speaker accent are not always valid indicators of the language for recognition, it is possible that listeners still do not exploit them to regulate the degree of language selectivity. The present design may reveal which of these two hypotheses is correct.

To summarize, we investigated whether L1 knowledge influences lexical access when listening to sentences in L2. With this aim, Dutch-English bilinguals completed an English lexical decision task on the last word of spoken sentences. In critical trials, this last word was either an interlingual homophone or a matched control word. To investigate the influence of sentence constraint, sentences were either low- or high-constraining. To test whether lexical access is sensitive to cues related to the native accent of the speaker, sentences were pronounced by a native Dutch speaker or by a native English speaker.

\section{Method}

\section{Participants}

Sixty-four students from Ghent University participated in the experiment for course credits or a monetary fee. All were native Dutch speakers and reported English as their L2. ${ }^{2}$ They started to learn English around the age of 14 years at secondary school, and because they were regularly

2 Although French is typically the second language of children raised in Flanders, we consider it here as the third language because our participants are much more proficient in English. So in this study, L2 is defined in terms of current dominance, and not of age of acquisition.
Table 1. Self-reported rating (seven-point Likert scale) of L1, L2, and L3 proficiency (standard deviations in parentheses).

\begin{tabular}{lll}
\hline \hline Language & \multicolumn{2}{c}{ Skill } \\
\hline & Writing & $5.78(0.87)$ \\
& Speaking & $5.85(0.99)$ \\
L1 (Dutch) & Reading & $6.12(0.82)$ \\
& Understanding & $6.35(0.74)$ \\
& General proficiency & $6.03(0.68)$ \\
& Writing & $4.71(1.01)$ \\
& Speaking & $4.98(0.99)$ \\
L2 (English) & Reading & $5.34(0.99)$ \\
& Understanding & $5.52(1.00)$ \\
& General proficiency & $5.03(0.85)$ \\
L3 (French) & General proficiency & $4.00(1.25)$ \\
\hline
\end{tabular}

exposed to their L2 through popular media, entertainment, and English university textbooks, they were all quite proficient in their L2, even though they lived in a clearly L1-dominant environment. ${ }^{3}$ After the experiment, participants were asked to rate their L1 (Dutch) and L2 (English) proficiency with respect to several skills (reading, writing, speaking, understanding and general proficiency) on a seven-point Likert scale ranging ( $1=$ very bad, $7=$ very good). We also assessed general L3 (French) proficiency. Means are reported in Table 1. Mean self-reported L1 $(M=6.03)$, L2 $(M=5.03)$, and L3 $(M=4.00)$ general proficiency differed significantly (dependent samples $t$-tests yielded $p \mathrm{~s}<.001$ ).

Participants were not informed that their L1 knowledge would be of any relevance to the experiment. Thirtythree participants listened to the sentences pronounced by the native Dutch speaker, 31 participants listened to the sentences pronounced by the native English speaker. One participant who made more than $20 \%$ errors was excluded from all analyses.

\section{Stimulus materials}

Target stimuli consisted of 240 stimuli: 30 interlingual Dutch-English homophones (e.g., lief "sweet" - leaf /liff/), 30 matched English control words, 60 English filler words, and 120 nonwords. All targets were selected

3 In a previous study in our lab, a comparable group of participants was asked to report their exposure level in Dutch, English, French and other languages. Participants reported that they are exposed to Dutch during $92 \%$ of the time, to English during $6 \%$ of the time, and to French during merely $1 \%$ of the time, which is almost negligible and not much more than for other languages such as German and Spanish (somewhat less than $1 \%$ of the time). 
from the stimulus list of Lagrou et al. (2011), in order to increase comparability across studies, and therefore make it possible to assess the context effects while keeping stimuli constant. Targets were between three and seven phonemes long, and control words were matched with these homophones with respect to number of phonemes and English frequency as reported in the CELEX database ( $p$ s > .32). Nonwords were created with the WordGen stimulus software (Duyck, Desmet, Verbeke \& Brysbaert, 2004). They were phoneme strings with no Dutch or English meaning, but with a legal English phonology, and they were matched with interlingual homophones and control words with respect to word length. For each target, a low- and high-constraining sentence was constructed, resulting in 480 sentences. Sentences were matched in terms of number of words and syntactic structure. Targets were always in the final position of the sentence. To ensure that participants would not see the same target twice, sentences were divided across two lists. The lowand high-constraining sentences for each homophonecontrol pair are included in the Supplementary Materials accompanying this paper on the online pages of the journal (http://journals.cambridge.org/BIL). Sentences were pronounced by a native Dutch speaker who was also a highly proficient English speaker, or by a native English speaker who was also a highly proficient Dutch speaker. Using WaveLab software, stimulus materials were recorded in a sound-attenuated booth by means of a SE Electronics USB1000A microphone with a sampling rate of $44.1 \mathrm{kHz}$ and a 16-bit sample size. Sentence and target durations were measured with WaveLab software.

\section{Sentence completion}

To verify the constraint manipulation of the sentences containing an interlingual homophone or control word, a sentence completion study was conducted with 20 further participants. Participants saw each sentence without the interlingual homophone/control word, and were instructed to complete the sentence with the first word that came to mind when reading the sentence. Production probabilities for interlingual homophones and control words were extremely low for low-constraining sentences, and were very high for high-constraining sentences. Production probabilities for the irrelevant L1 translation equivalents of the homophone were extremely low for low- and highconstraining sentences (see Table 2).

Additionally, another 15 participants were asked to rate the plausibility of the low-constraining sentences on a scale from 1 (not at all plausible) to 9 (very plausible). A paired $t$-test demonstrated that plausibility ratings for homophone sentences $(M=5.79, S D=0.50)$ did not differ from ratings for control word sentences $(M=6.06$, $S D=1.51), t(29)=-0.76, p=.46$.
Table 2. Production probabilities for interlingual homophones, control words, and L1 translation equivalents of the homophone in low- and high-constraining sentences (standard deviations in parentheses).

\begin{tabular}{lcc}
\hline \hline \multirow{2}{*}{ Word type } & \multicolumn{2}{c}{ Sentence constraint } \\
\cline { 2 - 3 } & Low & \multicolumn{1}{c}{ High } \\
\hline Interlingual homophone & $0.01(0.02)$ & $0.81(0.08)$ \\
Control word & $0.02(0.04)$ & $0.77(0.17)$ \\
L1 translation equivalent & $0.02(0.11)$ & $0.0008(0.0006)$ \\
\hline \hline
\end{tabular}

\section{Speakers}

The native Dutch speaker was a 25-year-old female with Dutch as L1 and English as L2. She had 12 years of L2 experience. Her English was very fluent but characterized by a clear Dutch accent. The native English speaker was a 45-year-old female with English as L1 and Dutch as L2. She had L2 experience since she moved to the Dutchspeaking part of Belgium 15 years ago. Her Dutch was very fluent but characterized by a clear English accent.

\section{Procedure}

Participants received written instructions in English (their L2) to perform an English lexical decision task on the last word of each sentence. They wore a headphone through which sentences were presented auditorily. Before the experiment, a practice session of 24 trials was completed. Each trial started with a $500 \mathrm{~ms}$ presentation of a fixation cross in the center of the screen. After another $200 \mathrm{~ms}$ the sentence was presented. Then participants had to decide whether the last word was an English word or a nonword. When a word (nonword) was presented, participants used their right (left) index finger to press the right (left) button of a response box. Visual feedback was presented on the screen during $200 \mathrm{~ms}$ (i.e., when an error was made the screen turned red, when the response was correct, “OK!" appeared). The next trial started $500 \mathrm{~ms}$ later. After the experiment, participants completed a questionnaire assessing self-ratings of $\mathrm{L} 1$ and L2 proficiency (reading, speaking, writing, understanding, and general proficiency), and general L3 proficiency on a seven-point Likert scale ( $1=$ very bad, $7=$ very good), and a backward translation test to verify that they knew the L2 words.

\section{Results}

On average, participants made $6.54 \%$ errors $(S D=2.30)$. Errors, trials with RTs faster than $300 \mathrm{~ms}$ after target onset, and trials with RTs more than 2.5 standard deviations 


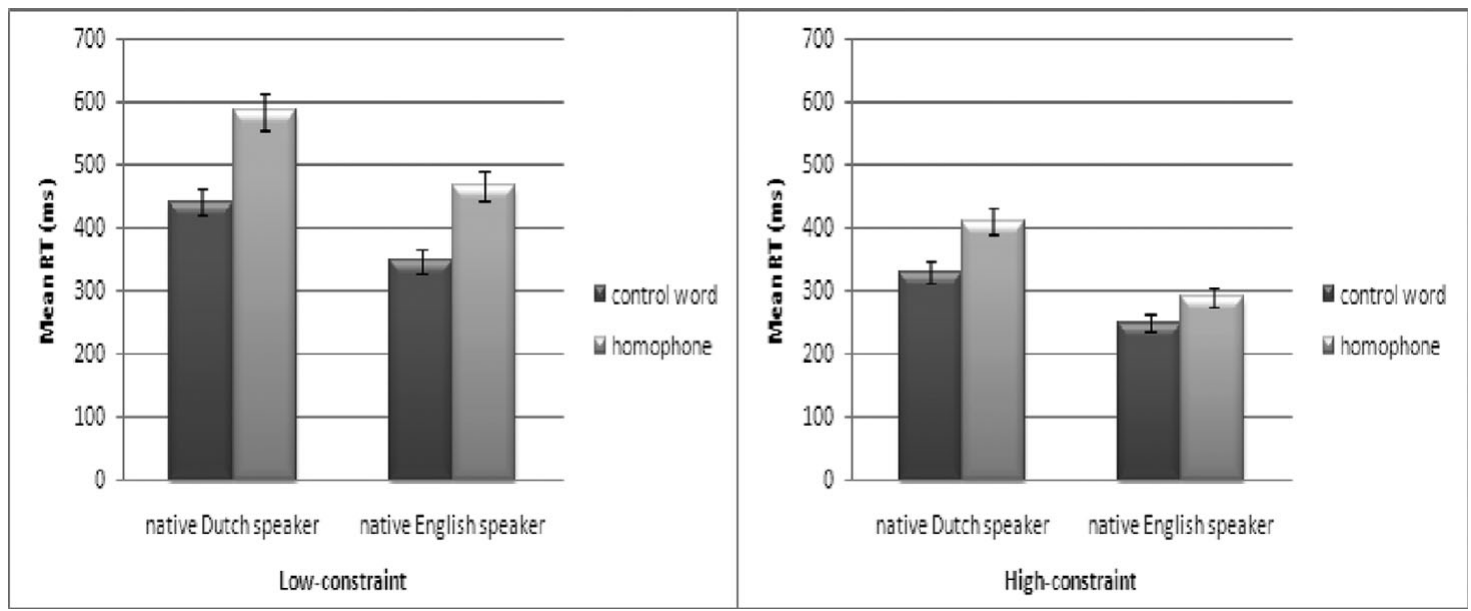

Figure 1. Reaction times (RTs) on homophones and matched control words as a function of sentence constraint (low vs. high) and native accent of the speaker (native Dutch vs. native English). The vertical bars represent the 95\% confidence interval.

Table 3. Mean pronunciation durations of target words (homophones and controls) as a function of constraint and speaker.

\begin{tabular}{lcc}
\hline \hline Speaker & Low-constraint & High-constraint \\
\hline Native Dutch speaker & 380 & 361 \\
Native English speaker & 470 & 442 \\
\hline \hline
\end{tabular}

above the participant's mean RT after target offset for word targets were excluded from the analyses. As a result, $8.29 \%$ of the data were excluded from the analyses. In all experiments, reported latency analyses are based on RTs measured from (auditory) target offset. ${ }^{4}$ We reported these measures because the native and non-native speaker differed in pronunciation duration $(p<.01)$. Importantly, the pronunciation durations did not differ systematically for low- and high-constraining sentences $(p>.15)$ (see Table 3).

An ANOVA on the reaction times (see Figure 1 and Table 4) with target type (interlingual homophone vs. control) and sentence constraint (low vs. high) as the independent within-subjects variables and speaker (native Dutch vs. native English) as the independent betweensubjects variable revealed a main effect of target type, $F 1(1,61)=234.50, p<.001, \mathrm{\eta}^{2}{ }_{p}=.79 ; F 2(1,29)=27.01$, $p<.001, \mathrm{y}^{2}{ }_{p}=.48$, indicating that reaction times were significantly slower on interlingual homophones than on control words. Importantly, the main effect of sentence constraint was significant, $F 1(1,61)=325.92, p<.001$, $\mathrm{\eta}^{2}{ }_{p}=.84 ; F 2(1,29)=152.36, p<.001, \mathrm{j}^{2}=.84$, indicating that participants responded significantly faster on

4 When latency analyses were based on reaction times measured from (auditory) target onset, the same pattern of results was obtained. targets that were preceded by a high-constraining sentence context than on targets that were preceded by a lowconstraining sentence context. This ensures validity of the constraint manipulation. The main effect of speaker was also significant, $F 1(1,61)=10.24, p<.01, \mathrm{\eta}^{2}{ }_{p}=.14$; $F 2(1,29)=80.23, p<.001, \mathrm{\eta}^{2} p=.74$, indicating that participants responded faster when the sentences were pronounced by the native English speaker than when they were pronounced by the native Dutch speaker. Moreover, the interaction between sentence constraint and target type was also significant, $F 1(1,61)=28.49$, $p<.001, \mathrm{\eta}^{2}=.32 ; F 2(1,29)=9.30, p<.01, \mathrm{y}^{2}=.24$, showing a larger homophone effect in the low-constrained condition. Planned comparisons demonstrated that the homophone effect was significant when the target was preceded by a low-constraining sentence, $F 1(1,61)=173.23, p<.001, \mathrm{n}^{2} p=.74 ; F 2(1,29)=46.68$, $p<.001, \mathrm{\eta}^{2} p=.62$, but also when the target was preceded by a high-constraining sentence, $F 1(1,61)=56.85$, $p<.001, \mathrm{\eta}^{2}{ }_{p}=.48 ; F 2(1,29)=6.32, p<.05, \mathrm{\eta}^{2} p=.18$. The interaction between target type and speaker was significant in the by-subjects analysis, $F 1(1,61)=6.84$, $p<.05, \mathrm{n}_{p}^{2}=.10 ; F 2(1,29)=3.06, p=.09, \mathrm{\eta}^{2}=.10$, with a larger effect for the Dutch native speaker. Planned comparisons demonstrated that the homophone effect was significant when sentences were pronounced by the native Dutch speaker, $F 1(1,61)=168.76, p<.001, \mathrm{\eta}^{2}{ }_{p}=.80$; $F 2(1,29)=23.26, p<.001, \mathrm{\eta}^{2}=.42$, but also when sentences were pronounced by the native English speaker, $F 1(1,61)=76.95, p<.001, \mathrm{y}^{2}{ }_{p}=.82 ; F 2(1,29)=21.10$, $p<.001, \mathrm{\eta}_{p}^{2}=.45$. No further interaction was significant, all $F 1 \mathrm{~s}<1, F 2 \mathrm{~s}<1$.

It is conceivable that the interactions of the interlingual homophone effect with semantic constraint and speaker accent are influenced by the overall faster reaction times on high-constraint sentences and sentences pronounced by 
Table 4. Mean RTs and effect (in milliseconds) as a function of word type, constraint and speaker.

\begin{tabular}{llccc}
\hline \hline Constraint & Speaker & Control words & Homophones & Effect \\
\hline Low-constraint & & & & \\
& Native Dutch speaker & 444 & 587 & 143 \\
& Native English speaker & 349 & 463 & 114 \\
High-constraint & & & & \\
& & 332 & 412 & 80 \\
& Native Dutch speaker & 248 & 285 & 37 \\
& Native English speaker & &
\end{tabular}

the native English speaker, yielding smaller homophone effects. On this account, semantic information and cues inherent to the native accent of the speaker speed up word recognition, but are not used as a strict cue to restrict lexical search to a single language, and therefore do not modulate the degree of language nonselectivity. To test this hypothesis taking into account baseline RT differences across constraint conditions, we first calculated the percentage homophone interference score for each semantic context. ${ }^{5}$ For each participant, and for both the low- and high-constraining sentences, the difference between the reaction times on homophone sentences and the reaction times on control sentences was divided by the reaction times on control sentences. A paired $t$-test demonstrated that this interference score was not significantly different for low- and high-constraining sentences, $p=.26$. Second, we also calculated the percentage homophone interference score for both the Dutch and the English speaker. Again, a paired $t$-test revealed that the interference score was not significantly different for both speakers, $p=.87$. This analysis supports the possibility that the interaction effects of the homophone effect with both semantic constraint and the native language of the speaker reflect overall RT differences. In any case, in each of the separate conditions, the homophone effect, as a marker of cross-lingual lexical interactions, was significant.

Because the results of the plausibility ratings of the low-constraining sentences demonstrated that some of the low-constraining sentences may not have been very plausible, we ran an additional analysis in which we excluded the low-constraining sentences and their high-constraining counterpart of which the homophone or control word had a plausibility score lower than 4 on a scale from 1 (not at all plausible) to 9 (very plausible). As a consequence, ten sentences were excluded from this analysis. (These sentences are marked in the Supplementary Materials with an asterisk.) However, the exclusion of these sentences did not change the pattern of results, except that the interaction between sentence

5 We thank an anonymous reviewer for this suggestion. constraint and target type was only significant in the analysis by subjects, $F 1(1,61)=15.23, p<.001, \mathrm{\eta}^{2}{ }_{p}=.28$ but not in the analysis by items, $F 2<1$, probably because this of course limited the number of critical stimuli considerably.

\section{Discussion}

The present study investigated whether lexical access is language-nonselective when listening to words that are embedded in meaningful sentences in L2. Furthermore, we examined whether the degree of language nonselectivity is modulated by the semantic constraint of the sentences and by the (native or nonnative) accent of the speaker of the sentences. DutchEnglish late bilinguals, immersed in an L1-dominant environment, completed an L2 auditory lexical decision task on the last word of low- and high-constraining sentences that were pronounced by a native Dutch or by a native English speaker. The results showed that reaction times were significantly slower on interlingual homophones (e.g., lief "sweet" - leaf /liif/) than on matched control words. This indicates that our bilingual listeners activated both the $\mathrm{L} 2$ and the $\mathrm{L} 1$ representation of the homophones, and it implies that sub-phonemic cues provided by the stream of speech in a sentence are not used to restrict lexical access to a single lexicon. We found this effect, even though the participants in this study were late bilinguals that are moderately proficient in their L2, and typically use it less than $5 \%$ of the time (for a quantification of language dominance in this homogenous population, see Duyck \& Warlop, 2009). A question that needs to be investigated in future research concerns whether these cross-lingual effects may interact with lower/higher L2 proficiency levels than those of the current study. The current results extend the monolingual finding of Frazier and Rayner (1990) for example, who reported that intralingual homophones are recognized more slowly than non-homophones. The present study also extends previous work on isolated auditory word recognition (e.g., Lagrou et al., 2011; Marian \& Spivey, 2003; Schulpen et al., 2003; Spivey \& Marian, 1999; 
Weber \& Cutler, 2004) to word recognition in more ecologically valid contexts, namely sentences.

Second, we considered the influence of factors potentially modulating cross-lingual activation spreading, namely semantic constraint of the sentence and speaker accent. The main effect of sentence constraint is consistent with earlier findings in monolingual and bilingual studies of visual word recognition. In the monolingual domain (e.g., Schwanenflugel \& LaCount, 1988; Schwanenflugel \& Shoben, 1985; Stanovich \& West, 1983) participants are typically faster to recognize words that are highly predictable from the preceding sentence context. In the bilingual visual domain, faster reading times for high-constraining sentences than for low-constraining sentences were also found, for example, by Van Assche et al. (2011). Here, we generalize this effect to auditory bilingual word recognition. Importantly, the interaction between the homophone effect and semantic constraint of the sentence was significant, which indicates that the homophone effect was smaller, but not completely annulled, when the preceding sentence context was highly constraining towards the target. This suggests that the semantic constraint of the sentence affects the activation level of representations from both the native and the nonnative language in the bilingual language system, but this activation pattern does not completely eliminate cross-language activation as such. Note that studies in the domain of visual word recognition show a mixed pattern of results, with some studies finding that semantic constraint eliminates cross-lingual effects (Schwartz \& Kroll, 2006; Van Hell \& de Groot, 2008) and other studies showing that such effects survive a highly semantically constraining sentence (Libben \& Titone, 2009; Titone et al., 2011; Van Assche et al., 2011). In the bilingual auditory domain, the results of Chambers and Cooke (2009) and FitzPatrick and Indefrey (2010) suggest that semantic constraints imposed by a sentence context may annul activation of non-target language lexical competitors. The findings from the present study, however, demonstrate that such a constraint may influence word recognition, but does not NECESSARILY eliminate cross-lingual lexical interactions. A possible explanation for these divergent results could be that we used interlingual homophones, of which the lexical and phonological overlap is maximal. In contrast with our stimuli, Chambers and Cooke used near-homophones and the stimuli of FitzPatrick and Indefrey only shared an overlapping onset. Hence, cross-lingual activation spreading in those studies was much weaker and therefore probably also more easily overridden by the stronger semantic context manipulation.

Third, we tested whether the homophone interference effect was modulated by the native accent of the speaker. The results showed that participants are faster when sentences are pronounced by the native English speaker than when they are pronounced by the native Dutch speaker. It is possible that the threshold for word recognition is exceeded faster when the pronunciation provides a closer match to the listener's stored representation, which is indeed the case when English sentences are pronounced by a native English speaker. ${ }^{6}$ This explanation is also compatible with the results of Adank, Evans, Stuart-Smith and Scott (2009), who demonstrated that listeners have difficulties processing speech with a nonnative accent. At least, the fact that reaction times are influenced by the native accent of the speaker demonstrates that the different accents of our speakers indeed contained language-specific acoustic information, which constitutes a valid manipulation check for the assumed sub-phonemic differences between languages. In future research, it would be interesting to investigate more in detail whether this accent-effect arises because Dutch speech increases the salience of Dutch or the fact that accented speech is less intelligible overall. The results also showed that the homophone effect was reduced (but not eliminated) when sentences were pronounced by the native English speaker. This suggests that sub-phonemic cues, inherent to the speaker's L1 are used to some extent as a cue to restrict lexical search to a single lexicon. These findings are consistent with Schulpen et al. (2003), who reported that the English pronunciation of (auditorily presented) interlingual homophones led to stronger priming of the English target than the Dutch pronunciation of that same homophone. They are also partly consistent with Ju and Luce (2004), who found that L1 recognition (Spanish) was influenced by L2 (English) competitors if L1 materials contained L2 sub-phonemic features (i.e., English VOTs), even though the strong acoustic feature (i.e., voicing) in that study was manipulated systematically, whereas the present stimuli differed on a wider range of acoustic parameters, so that such information is less reliable as a cue for lexical selection.

These findings have several theoretical implications. First, this study demonstrates that the languagenonselective nature of lexical access is not fundamentally altered by the preceding (low-constraining) sentence context: even unilingual language context is not used as a restrictive lexical cue, even though this might be an efficient strategy to speed up word recognition as this would surely eliminate a sizable proportion of the considered lexical candidates. Note, however, that Vitevitch (2012) conducted a corpus analysis which challenges the fact that many lexical candidates are active at the same time.

6 For these participants, L2 comprehension is typically more frequent than L2 production. And, because most L2 exposure originates from media, television, music, etc., participants are more exposed to speech produced by native speakers than by Flemish (Dutch) speakers. 
Second, the current results show that a highconstraining sentence context does influence the language nonselectivity of lexical access in the bilingual language system. Nevertheless, it does not prevent activation of lexical representations in the non-target language, not even when these representations do not meet these semantic restrictions (the critical stimuli were interlingual homophones and therefore only have form overlap across languages).

Third, the results of the present study also demonstrate that speech-specific cues provided by the native accent of the speaker are used to some extent to modulate the language-nonselective nature of bilingual lexical access. However, the fact that the homophone effect remained significant when sentences were pronounced by the native Dutch speaker demonstrates that these sub-phonemic cues are not applied to completely restrict lexical access to the currently relevant lexicon.

Our interlingual homophone effects can be explained by extending monolingual models of auditory word recognition such as the Distributed Model of Speech Perception (Gaskell \& Marslen-Wilson, 1997), NAM (Luce \& Pisoni, 1998), Shortlist (Norris, 1994; Norris, McQueen, Cutler \& Butterfield, 1997), and TRACE (Elman \& McClelland, 1988; McClelland \& Elman, 1986) if they are extended with the assumption that L2 representations are part of the same system as, and interact with, L1 representations. The results of the present study also demonstrate that there is an influence of top-down factors such as the semantic constraint of the sentence or sub-phonemic information provided by the native accent of the speaker, to inhibit lexical representations belonging to a particular language. Thus, at a theoretical level, the results of the present study are compatible with a model of bilingual auditory word recognition that supports language-nonselective bottom-up activation with a role for top-down connections that does not result in a functionally language-selective system. Because the homophone effect was reduced, but did not disappear in the high constraining condition and in the condition in which sentences were pronounced by the native English speaker, we can conclude that this role is limited. These findings are partly in line with the visual domain, for which there is a dominant model of bilingual word recognition, i.e., the BIA+ model (Dijkstra \& Van Heuven, 2002). This model consists of language nodes which act as language membership representations within the word identification system, but these nodes do not have topdown connections that regulate cross-lingual activation.

In sum, the present study provides evidence for the conclusion that lexical access is languagenonselective. However, when the semantic context is highly constraining and when the native accent of the speaker is compatible with the target language, crosslingual interactions are reduced (but not eliminated) by these semantic and accent-specific cues when listening to sentences in L2.

\section{References}

Adank, P., Evans, B. G., Stuart-Smith, J., \& Scott, S. K. (2009). Comprehension of familiar and unfamiliar native accents under adverse listening conditions. Journal of Experimental Psychology: Human Perception and Performance, 35, 520529.

Binder, K., \& Rayner, K. (1998). Contextual strength does not modulate the subordinate bias effect: Evidence from eyefixations and self-paced reading. Psychonomic Bulletin \& Review, 5, 271-276.

Chambers, C. G., \& Cooke, H. (2009). Lexical competition during second-language listening: Sentence context, but not proficiency, constrains interference from the native lexicon. Journal of Experimental Psychology: Learning, Memory, and Cognition, 35, 1029-1040.

Dijkstra, T., \& Van Heuven, W. J. B. (1998). The BIA model and bilingual word recognition. In J. Grainger \& A. M. Jacobs (eds.), Localist connectionist approaches to human cognition, pp. 189-225. Mahwah, NJ: Lawrence Erlbaum.

Dijkstra,T., \& Van Heuven, W. J. B. (2002). The architecture of the bilingual word recognition system: From identification to decision. Bilingualism: Language and Cognition, 5, 175197.

Duyck, W. (2005). Translation and associative priming with cross-lingual pseudohomophones: Evidence for nonselective phonological activation in bilinguals. Journal of Experimental Psychology: Learning, Memory, and Cognition, 31, 1340-1359.

Duyck, W., Desmet, T., Verbeke, L. P. C., \& Brysbaert, M. (2004). WordGen: A tool for word selection and nonword generation in Dutch, English, German, and French. Behavior Research Methods, Instruments, \& Computers, 36, 488-499.

Duyck, W., Van Assche, E., Drieghe, D., \& Hartsuiker, R. J. (2007). Visual word recognition by bilinguals in a sentence context: Evidence for nonselective lexical access. Journal of Experimental Psychology: Learning, Memory, and Cognition, 33, 663-679.

Duyck, W., \& Warlop, N. (2009). Translation priming between the native language and a second language: New evidence from Dutch-French bilinguals. Experimental Psychology, 56, 173-179.

Elman, J. L., \& McClelland, J. L. (1988). Cognitive penetration of the mechanisms of perception: Compensation for coarticulation of lexically restored phonemes. Journal of Memory and Language, 27, 143-165.

FitzPatrick, I., \& Indefrey, P. (2010). Lexical competition in nonnative speech comprehension. Journal of Cognitive Neuroscience, 22, 1165-1178.

Frazier, L., \& Rayner, K. (1990). Taking on semantic commitments: Processing multiple meanings versus multiple senses. Journal of Memory and Language, 29, 181-200.

Gaskell, M. G., \& Marslen-Wilson, W. D. (1997). Integrating form and meaning: A distributed model of speech 
perception. Language and Cognitive Processes, 12, 613656.

Ju, M., \& Luce, P. A. (2004). Falling on sensitive ears Constraints on bilingual lexical activation. Psychological Science, 15 (5), 314-318.

Lagrou, E., Hartsuiker, R. J., \& Duyck, W. (2011). Knowledge of a second language influences auditory word recognition in the native language. Journal of Experimental Psychology: Learning, Memory, and Cognition, 37, 952-965.

Lee, L., \& Nusbaum, H. C. (1993). Processing interactions between segmental and suprasegmental information in native speakers of English and Mandarin Chinese. Perception \& Psychophysics, 53, 175-165.

Libben, M. R., \& Titone, D. A. (2009). Bilingual lexical access in context: Evidence from eye movements during reading. Journal of Experimental Psychology: Learning, Memory, and Cognition, 35, 381-390.

Luce, P. A., \& Pisoni, D. B. (1998). Recognizing spoken words: The neighborhood activation model. Ear and Hearing, 19, $1-36$.

Marian, V., \& Spivey, M. (2003). Bilingual and monolingual processing of competing lexical items. Applied Psycholinguistics, 24, 173-193.

McClelland, J. L., \& Elman, J. L. (1986). The TRACE model of speech perception. Cognitive Psychology, 18, 1-86.

Norris, D. G. (1994). Shortlist: A connectionist model of continuous speech recognition. Cognition, 52, 189234.

Norris, D. G., McQueen, J. M., Cutler, A., \& Butterfield, S. (1997). The possible-word constraint in the segmentation of continuous speech. Cognitive Psychology, 34, 191-243.

Onifer, W., \& Swinney, D. A. (1981). Accessing lexical ambiguities during sentence comprehension: Effects of frequency and contextual bias. Memory \& Cognition, 9, 225-236.

Rayner, K., \& Frazier, L. (1989). Selection mechanisms in reading lexically ambiguous words. Journal of Experimental Psychology: Learning, Memory, and Cognition, 15, 779-790.

Schulpen, B., Dijkstra, T., Schriefers, H. J., \& Hasper, M. (2003). Recognition of interlingual homophones in bilingual auditory word recognition. Journal of Experimental
Psychology: Human Perception and Performance, 29, 1155-1178.

Schwanenflugel, P. J., \& LaCount, K. L. (1988). Semantic relatedness and the scope of facilitation four upcoming words in sentences. Journal of Experimental Psychology: Learning, Memory, and Cognition, 14, 344-354.

Schwanenflugel, P. J., \& Shoben, E. J. (1985). The influence of sentence constraint on the scope of facilitation for upcoming words. Journal of Memory and Language, 24, 232-252.

Schwartz, A. I., \& Kroll, J. F. (2006). Bilingual lexical activation in sentence context. Journal of Memory and Language, 55, 197-212.

Spivey, M. J., \& Marian, V. (1999). Cross talk between native and second languages: Partial activation of an irrelevant lexicon. Psychological Science, 10, 281-284.

Stanovich, K. E., \& West, R. F. (1983). On priming by a sentence context. Journal of Experimental Psychology: General, $112,1-36$.

Titone, D., Libben, M., Mercier, J., Whitford, V., \& Pivneva, I. (2011). Bilingual lexical access during L1 sentence reading: The effects of L2 knowledge, semantic constraint, and L1-L2 intermixing. Journal of Experimental Psychology: Learning, Memory, and Cognition, 37, 1412-1431.

Van Assche, E., Drieghe, D., Duyck, W., Welvaert, M., \& Hartsuiker, R. J. (2011). The influence of semantic constraints on bilingual word recognition during sentence reading, Journal of Memory and Language, 64, 88-107.

Van Assche, E., Duyck, W., Hartsuiker, R. J., \& Diependaele, K. (2009). Does bilingualism change native-language reading? Cognate effects in a sentence context. Psychological Science, 20, 923-927.

Van Hell, J. G., \& de Groot, A. M. B. (2008). Sentence context modulates visual word recognition and translation in bilinguals. Acta Psychologica, 128, 431-451.

Vitevitch, M. (2012). What do foreign neighbors say about the mental lexicon? Bilingualism: Language and Cognition, 15, 167-172.

Weber, A., \& Cutler, A. (2004). Lexical competition in nonnative spoken-word recognition. Journal of Memory and Language, 50, 1-25. 